GOVDOC
BRA
3819

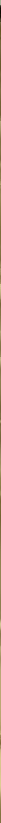

An Inventory of the Coastal Resources of the Commonwealth of Massachusetts

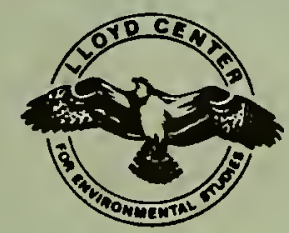

Lloyd Center

For

Environmental

Studies
Proporty ut


Library

Massachusetts

Coastal Zone

Management

Program 
Digitized by the Internet Archive in 2011 with funding from Boston Public Library

http://www.archive.org/details/barrierbeachessa00lloy 


\section{BARRIER BEACHES, SALT MARSHES, AND TIDAL FLATS \\ An Inventory of the Coastal Resources of the Commonwealth of Massachusetts}

by

Alan Lee Hankin

Lucille Constantine

Steve Bliven

January 1985

THE LLOYD CENTER FOR ENVIRONMENTAL STUDIES

and

THE MASSACHUSETTS COASTAL ZONE MANAGEMENT PROGRAM

with major assistance from

The United States Department of the Interior Fish and Wildlife Service, Region 5

Publication: 非13899-27-600-1-85 C.R.

Approved by: Daniel Carter, State Purchasing Agent

Commonwealth of Massachusetts, Michael S. Dukakis, Governor Executive Office of Environmental Affairs, James S. Hoyte, Secretary

Coastal Zone Management Program, Richard F. Delaney, Di rector 


\section{ACKNOWLEDGEMENTS}

Preparation and publication of this document has involved the efforts of several people and organizations. The Lloyd Center and the Massachusetts Coastal Zone Management Program would like to express their special appreciation to the following for their efforts in the planning, research, data collection, and support of this project:

- Ralph Tiner and John Organ of the US Department of the Interior, Fish and Wildlife Service, Region $V$. These two gentlemen made available raw data, maps, equipment, and facilities, as well as their time and expertise to develop the data contained herein, and reviewed drafts of this report. Without their efforts, this project would not have been possible.

- Lloyd Center researchers and volunteers David Jansen, Becky Goldstein, and Lisa Caron reviewed preliminary work and the resulting data for accuracy and clarity.

- Maronn W. Sternack did the typing and provided the usual cogent comments.

While many of the better features of this report came from the above sources, any flaws remain the sole responsibility of the authors, who would appreciate being made aware of them.

Alan Lee Hankin

Lucille Constantine

Lloyd Center for Environmental Studies

430 Potomska Road

South Dartmouth, MA 02748

Steve Bliven

Massachusetts Coastal Zone Management Office 100 Cambridge Street, 20th Floor

Boston, MA 02202 
TABLE OF CONTENTS

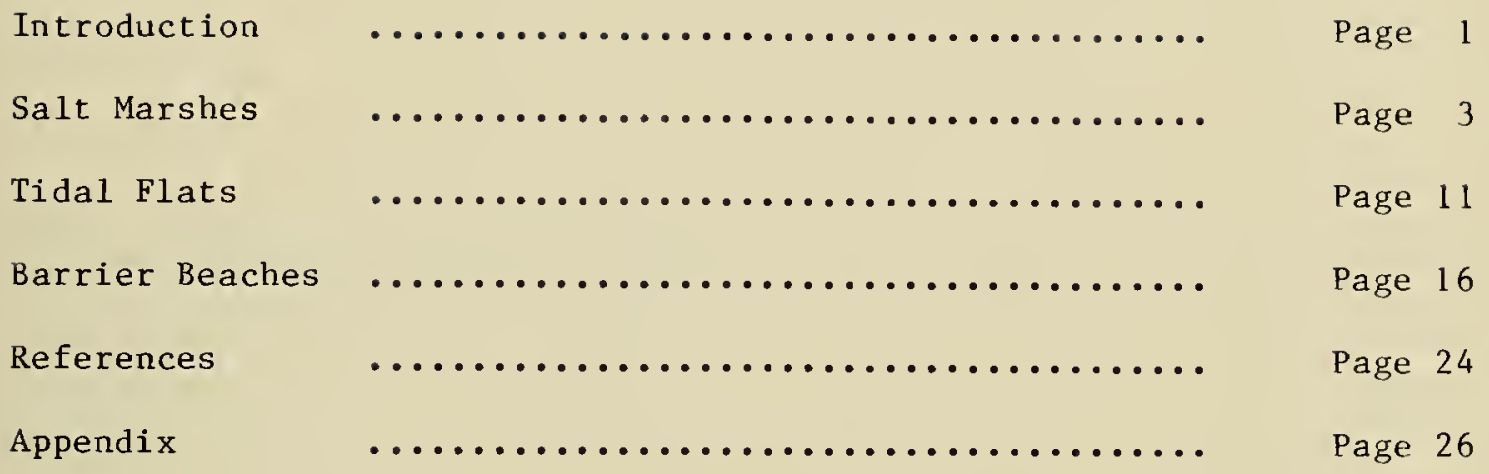

\section{TABLES OF DATA}

Table 1

Table 2

Table 3

Table 4

Table 5

Table 6

Table 7

Table 8

Table 9

Table 10
State and County Acreage of Three Major Natural Resources

Salt Marsh Acreage within Counties

Salt Marsh Acreage by Town

Comparison of Salt Marsh Acreages in Prior Studies

Tidal Flat Acreage within Counties

Tidal Flat Acreage by Town

Numbers of Barrier Beach Segments and Acreage within Counties

Barrier Beach Acreage by Town

Size Distribution of Massachusetts Barrier Beaches

The 30 Largest Barrier Beach Landforms in Massachusetts
Page 2

Page 6

Page 8

Page 10

Page 12

Page 14

Page 18

Page 20

Page 22

Page 23 



\section{INTRODUCTION}

Cities and towns throughout the Commonwealth of Massachusetts have been charged with the primary responsibility for the management of local wetlands resources, particularly through the administration and enforcement of the Massachusetts Wetlands Protection Act (MGL C.131 s. 40). Although some political divisions have inventoried the wetlands within their borders, most do not have quantified information on the amount of resources under their protection. Local

Conservation Commissions and Planning Boards are therefore often unaware of the extent of salt marsh, barrier beach, and tidal flat acreage within their jurisdiction. Additionally, the regional, county, and state agencies charged with developing broader scale planning, resource protection, and enforcement activities often have difficulty setting priorities for their work. By providing basic information on the extent of some of these coastal wetland areas, this report seeks to address these problems.

The following report will present a quantification of three of the most important coastal wetland resources in the Commonwealth; salt marshes, tidal flats, and barrier beaches. For each of these resource areas the report presents a brief introduction to the resource, a discussion of the methodology used in determining the acreage, a discussion of the results, and various tables to present the data. It is hoped that this study will lead to additional work further defining and analyzing these important resource areas.

For further information about the details of this project, data for individual communities, or further projects, please contact the Lloyd Center for Environmental Studies. 
Table 1

STATE AND COUNTY ACREAGE OF THREE MAJOR NATURAL RESOURCES

$\begin{array}{llll} & \text { Barrier Beaches } & \text { Salt Marshes } & \text { Tidal Flats } \\ \text { Massachusetts } & 18,888.0 & 48,104.6 & 41,514.2\end{array}$

Barnstable County
Bristol County
Dukes County
Essex County
Middlesex County
Nantucket County
Norfolk County
Plymouth County
Suffolk County

$$
\begin{array}{r}
8,723.1 \\
1,008.1 \\
2,135.7 \\
2,955.4 \\
0.0 \\
1,841.7 \\
95.7 \\
1,933.8 \\
194.5
\end{array}
$$

$$
\begin{array}{r}
15,201.0 \\
3,748.0 \\
1,027.7 \\
18,026.9 \\
0.0 \\
657.3 \\
1,056.7 \\
7,400.0 \\
987.0
\end{array}
$$

$$
\begin{array}{r}
17,808.6 \\
3,130.5 \\
1,258.7 \\
6,087.7 \\
0.0 \\
136.9 \\
2,334.6 \\
9,369.9 \\
1,387.3
\end{array}
$$

A11 areas given in acres 


\section{SALT MARSHES}

\section{Introduction}

Salt marshes are generally typified by flat, open, grassy areas along tidal waters. They are almost always found in sites protected from the high energy of the open coast; estuaries, salt ponds, or low, entrapped portions of barrier beaches. In Massachusetts' envi ronmental regulations, salt marshes are defined as coastal wetlands "that extend landward up to the highest high tide line, that is the highest spring tide of the year, and are characterized by plants that are well adapted to, or prefer living in, saline soils. Dominant plants within salt marshes are salt meadow cord grass (Spartina patens) and/or salt marsh cord grass (Spartina alterniflora)." (From the Coastal regulations to the Massachusetts Wetlands Protection Act, MGL C.131, s.40.) Other plant species often found within a salt marsh may include spikegrass (Distichlis spicata), marsh elder (Iva frutescens), saltworts (Salicornia sp.), and sea lavender (Limonium carolinanum).

Historically viewed as wasted land, salt marshes are now valued for their resource features. They provide wildlife habitat, produce and export large quantities of plant material to nearby waters to form the base of the marine food chain, lessen the effects of storms, take up some water-borne contaminants, and protect ground water from salt intrusion by forming a peat "dam" along the shore. A discussion of salt marsh ecology and resource values may be $f$ ound in The Ecology of New England High Salt Marshes: A community profile (1).

\section{Study Methodology}

Acreage of individual salt marsh segnents was taken from National Wetlands Inventory (NWI) maps provided by the US Fish and Wildlife Service (FWS) through their Region $\mathrm{V}$ office. These maps are based on US Geological Survey $71 / 2$ minute quadrangle topographic sheets. The wetlands boundaries delineated on these maps were prepared through analysis of aerial photography taken in April of 1977. Classification was based on vegetation, visible hydrology, and geography in accordance with Classification of Wetlands and Deepwater Habitats (2). A11 marshes over one-half acre in size are identified and located on the maps. Appendix I explains the classification scheme. For the purposes of this study, all areas classified as Estuarine Intertidal Emergent (E2EM) were considered to be salt marsh, notwithstanding any further subclassification. Areas classified as Estuarine Intertidal Scrub/shrub (E2SS) were listed as shrub marsh.

Each polygon representing E2EM vegetation was located on black1ine paper copies from mylar originals. Acreage was measured by tracing each polygon with a Numonics digital read-out planimeter. Each polygon was traced at least twice to ensure accuracy. Data were recorded by quadrangle, city or town, and county. County and state totals are presented in Table l. Acreages by town within county are found in Table 2. Table 3 lists acreages for all communities in the Commonwealth in sequential order; those communities with the most salt marsh are at the top, those with the least at the bottom. 
Comparison with previous measurements

In the past 30 years several measurements have been made of salt marsh acreages in Massachusetts. Results have varied depending on several factors, including:

1- Year of measurement

Strong regulatory protection of salt marshes was not enacted until 1963

(the Hatch Act and the Coastal Wetlands Restriction Act) and the current

high level of protection under the Coastal Regulations of the Wetlands

Protection Act was not implemented until 1978. Prior to this, marshes

were still being dredged or filled in significant increments.

2- Methodology of areal measurement

The areas of mapped salt marsh units have been calculated using the cell method (dividing the unit into squares of a known area; cells less than half "full" are ignored, those more than half "full" are considered equal to the known area), or by the use of planimeters of varying accuracy.

3- Differences in base map interpretation

As discussed below, different methods of aerial photo interpretation and different type and scale of base maps have been used.

4- Different levels of discrimination of polygon size Depending on the purpose of the study, different thresholds for minimum marsh unit size have been used, ranging from $1 / 2$ acre to 40 acres.

A brief description of the various prior surveys is provided below. For additional details the reader is directed to the original sources listed under References on Page 24.

1- MacConnel1 Mapdown (3)

Using aerial photography from $1951 / 1952$ and $1971 / 1972$, William MacConnel1 and his colleagues at the the University of Massachusetts in Amherst mapped the land use of the entire state. Included in the list of 100 categories were tidal marsh (defined as flooded twice daily, primarily Spartina alterniflora), irregularly flooded salt marsh (flooded at monthly high tides and by storms, primarily Spartina patens and Distichlis spicata), and salt meadows ditched for mosquito control. MacConnell and his staff made their interpretations of vegetacion and land use from 1:20,000 scale black and white aerial photography. In 1951 the minimum land area mapped was 10 acres, for 1971 better photography allowed this to be reduced to 3 acres. A cell, or dot-grid, method was used to calculate areas.

2- US Fish and Wildlife Service Two different types of study that included salt marsh areas have been done by the FWS. In 1954, an evaluation of those Massachusetts wetlands areas significant to waterfowl was done through the office of River Basin Studies (4). Using US Geological Survey $71 / 2$ minute quadrangle 
topographic sheets, wetland areas of 40 acres or more were identified, delineated, and measured using either a planimeter or the cell method. Figures were provided for both high (Spartina patens and Distichlis spicata) marshes and low (Spartina alterniflora) marshes.

A second study was done in 1954 and repeated in 1959 and 1964 to determine vulnerability of wetlands and changes in wetlands areas (5). The minimum threshold size was not stated in the report, but it may have been the same 40 acres as in the above study. The report estimates that $90 \%$ of the wetlands in Massachusetts were surveyed. A planimeter was used to measure areas.

Table 4 compares the results of these studies at the county and state levels with the results of the work at hand. It is the feeling of the authors that the present study, using recent, accurate mapping, interpretive, and measurment techniques provides the most up-to-date, comprehensive definition of Massachusetts' salt marshes.

\section{Discussion}

Almost $70 \%$ of Massachusetts' $48,104.6$ acres of salt marsh are found in Essex (37.5\%) and Barnstable (32\%) Counties. In Essex County, marshes make up over $10 \%$ of the total land area and within several towns the percentage is significantly higher. The town of Newbury is 30\% marsh (4,646 acres), Salisbury is $25 \%$ marsh $(2,536$ acres $)$, Essex is $24 \%$ marsh $(2,188$ acres $)$, and Ipswich is $21 \%$ marsh $(4,376$ acres).

Aptly named Marshfield on the south shore is $13 \%$ marsh (2,312 acres) and on Cape Cod (Barnstable County), Orleans and Eastham are each 15\% marsh ( 1377 and 1376 acres respectively) while Barnstable's 4,085 acres of marsh make up $10 \%$ of the town. 
Table 2

SALT MARSH ACREAGE WITHIN COUNTIES

Town

Barnstable County

\begin{tabular}{lr} 
Barnstable & $40,153.6$ \\
Orleans & $9,081.6$ \\
Eastham & $9,344.0$ \\
Yarmouth & $16,339.2$ \\
Dennis & $14,016.0$ \\
Sandwich & $27,916.8$ \\
Chatham & $10,438.4$ \\
Wellfleet & $13,324.8$ \\
Harwich & $14,342.4$ \\
Falmouth & $29,260.8$ \\
Brewster & $16,102.4$ \\
Mashpee & $16,614.4$ \\
Provincetown & $5,600.0$ \\
Bourne & $26,585.6$ \\
Truro Totals & $13,824.0$ \\
\hline & $262,944.0$
\end{tabular}

Bristol County

Dartmouth

Westport

Fairhaven

Swansea

Dighton

Freetown

Berkley

Somerset

Rehoboth

Seekonk

Acushnet

Fall River

New Bedford
Town Area

in Acres
$2 !, 371.2$

$12,691.2$
Salt Marsh acreage in

Town

$4,085.1$

$1,377.0$

$1,376.2$

$1,230.0$

$1,138.9$

$1,128.2$

$1,117.1$

$1,039.5$

558.2

529.4

420.5

337.5

332.6

297.7

$\frac{233.1}{15,201.0}$
Shrub Marsh acreage in

Town

0.0

0.0

9.3

0.0

5.5

0.0

7.4

2.4

0.0

0.0

0.0

0.0

0.0

0.0

$\frac{0.0}{24.6}$

$1,143.1$

$1,116.6$

607.5

184.8

151.4

145.8

125.9

97.3

88.5

52.8

30.4

3.9

0.0

$3,748.0$

504.1

Gay Head

$\frac{17,075.2}{71,788.8}$
241.1

89.7

89.3

53.6

29.0

$4,057.6$

$18,720.0$

$8,288.0$

$4,640.0$

$4,825.6$

20.9
0.0

0.0

0.0

0.0

0.0

0.0

0.0

0.0

0.0

0.0

0.0

0.0

$\frac{0.0}{0.0}$
West Tisbury
0.0

0.0

0.0

2.7

0.0

6.6

$\frac{0.0}{9.3}$ 
Essex County

Newbury

Ipswich

Salisbury

Essex

Rowley

Gloucester

Saugus

Newburyport

Rockport

Danvers

Nahant

Salem

Beverly

Manchester

Lynn

Peabody

Amesbury

Marblehead

Swampscott
$21,344.0$

$10,323.2$

$9,203.4$

$12,179.2$

$16,928.0$

$7,411.2$

$5,702.4$

$4,531.2$

$8,857.6$

678.4

$5,235.2$

$9,830.4$

4, 940.8

$7,174.4$

$10,758.4$

$8,838.4$

$2,828.8$

Totals $\frac{1,984.0}{164,326.6}$
$15,577.6$

$4,669.5$

$4,376.0$

$2,535.5$

$2,188.3$

$1,983.3$

$1,188.1$

670.4

179.9

48.2

34.9

32.8

30.8

29.5

19.1

17.6

9.0

8.0

6.0

0.0

$18,026.9$

0.0

0.0

$2,400.0$

$32,217.6$

657.3

3.7

Nantucket

Norfolk County
Quincy

Weymouth

Milton

Braintree

$10,649.6$

$6,438.4$

$11,340.8$

$8,448.0$

Totals $\frac{9,222.4}{46,099.2}$

Plymouth County

Marshfield
Scituate
Duxbury
Wareham
Norwell
Mattapoiset
Marion
Plymouth
Pembroke
Hull
Hingham
Kingston
Hanover

$18,252.8$

$10,924.8$

$15,686.4$

$24,339.2$

$13,651.2$

$11,187.2$

$9,152.0$

$66,048.0$

$14,886.4$

$1,619.2$

$14,457.6$

$12,179.2$

Totals $\frac{10,003.2}{222,387.2}$

\begin{tabular}{rr}
587.2 & 0.0 \\
158.4 & 0.0 \\
157.2 & 0.0 \\
147.9 & 0.0 \\
6.0 & 0.0 \\
\hline 056.7 & 0.0
\end{tabular}

$2,311.9$

$1,245.2$

$1,093.0$

917.0

462.5

349.9

305.8

290.5

145.4

89.6

89.2

83.8

$\frac{16.2}{7,400.0}$

0.0

0.0

1.7

0.0

0.0

0.0

0.0

0.0

0.0

0.0

3.7

0.0

$\frac{0.0}{5.4}$

Suffolk County

Revere

Boston

Winthrop

Chelsea
4, 044.8

$29,056.0$

$1,043.2$

$1,388.8$
490.6

391.7

102.7

2.0

987.0
0.0

0.0

0.0

$\frac{0.0}{0.0}$ 
Table 3

SALT MARSH ACREAGE BY TOWN (arranged in descending order)

\begin{tabular}{|c|c|c|c|c|}
\hline Town & County & Town Area & Sa1t Marsh & Shrub Marsh \\
\hline Newbury & ES & $15,577.6$ & $4,669.5$ & 6.6 \\
\hline Ipswich & ES & $21,344.0$ & $4,376.0$ & 2.2 \\
\hline Barnstable & BA & $40,153.6$ & $4,085.1$ & 0.0 \\
\hline Salisbury & ES & $10,323.2$ & $2,535.5$ & 0.0 \\
\hline Marshfield & PL & $18,252.8$ & $2,311.9$ & 0.0 \\
\hline Essex & ES & $9,203.4$ & $2,188.3$ & 0.0 \\
\hline Rowley & ES & $12,179.2$ & $1,983.3$ & 0.0 \\
\hline Orleans & $\mathrm{BA}$ & $9,081.6$ & $1,377.0$ & 0.0 \\
\hline Eastham & $\mathrm{BA}$ & $9,344.0$ & $1,376.2$ & 9.3 \\
\hline Scituate & PL & $10,924.8$ & $1,245.2$ & 0.0 \\
\hline Yarmouth & $\mathrm{BA}$ & $16,339.2$ & $1,230.0$ & 0.0 \\
\hline Gloucester & $\mathrm{ES}$ & $16,928.0$ & $1,188.1$ & 2.7 \\
\hline Dartmouth & $\mathrm{BR}$ & $39,558.4$ & $1,143.1$ & 0.0 \\
\hline Dennis & BA & $14,016.0$ & $1,138.9$ & 5.5 \\
\hline Sandwich & BA & $27,916.8$ & $1,128.2$ & 0.0 \\
\hline Chatham & BA & $10,438.4$ & $1,117.1$ & 7.4 \\
\hline Westport & BR & $35,353.6$ & $1,116.6$ & 0.0 \\
\hline Duxbury & PL & $15,686.4$ & $1,093.0$ & 1.7 \\
\hline Wellfleet & BA & $13,324.8$ & $1,039.5$ & 2.4 \\
\hline Wareham & $\mathrm{PL}$ & $24,339.2$ & 917.0 & 0.0 \\
\hline Saugus & ES & $7,411.2$ & 670.4 & 0.0 \\
\hline Nantucket & NA & $32,217.6$ & 657.3 & 3.7 \\
\hline Fairhaven & $B R$ & $7,936.0$ & 607.5 & 0.0 \\
\hline Quincy & No & $10,649.6$ & 587.2 & 0.0 \\
\hline Harwich & BA & $14,342.4$ & 558.2 & 0.0 \\
\hline Falmouth & BA & $29,260.8$ & 529.4 & 0.0 \\
\hline Edgartown & DU & $18,720.0$ & 504.1 & 0.0 \\
\hline Revere & SU & $4,044.8$ & 490.6 & 0.0 \\
\hline Norwe11 & PL & $13,651.2$ & 462.5 & 0.0 \\
\hline Brewster & $\mathrm{BA}$ & $16,102.4$ & 420.5 & 0.0 \\
\hline Boston & $\mathrm{SU}$ & $29,056.0$ & 391.7 & 0.0 \\
\hline Mattapoiset & PL & $11,187.2$ & 349.9 & 0.0 \\
\hline Mashpee & BA & $16,614.4$ & 337.5 & 0.0 \\
\hline Provincetown & $\mathrm{BA}$ & $5,600.0$ & 332.6 & 0.0 \\
\hline Marion & $\mathrm{PL}$ & $9,152.0$ & 305.8 & 0.0 \\
\hline Bourne & $\mathrm{BA}$ & $26,585.6$ & 297.7 & 0.0 \\
\hline Plymouth & PL & $66,048.0$ & 290.5 & 0.0 \\
\hline Chilmark & DU & $14,182.4$ & 241.1 & 0.0 \\
\hline Truro & $\mathrm{BA}$ & $13,824.0$ & 233.1 & 0.0 \\
\hline Swansea & BR & $14,585.6$ & 184.8 & 0.0 \\
\hline
\end{tabular}


SALT MARSH ACREAGE BY TOWN (continued)

\begin{tabular}{|c|c|c|c|c|}
\hline Town & County & Town Area & Salt Marsh & Shrub Marsh \\
\hline Newburyport & ES & $5,702.4$ & 179.9 & 0.0 \\
\hline Cohasset & NO & $6,438.4$ & 158.4 & 0.0 \\
\hline Weymouth & NO & $11,340.8$ & 157.2 & 0.0 \\
\hline Dighton & $B R$ & $14,304.0$ & 151.4 & 0.0 \\
\hline Milton & NO & $8,448.0$ & 147.9 & 0.0 \\
\hline Freetown & $B R$ & $23,174.4$ & 145.8 & 0.0 \\
\hline Pembroke & PL & $14,886.4$ & 145.4 & 0.0 \\
\hline Berkley & $\mathrm{BR}$ & $10,496.0$ & 125.9 & 0.0 \\
\hline Winthrop & SU & $1,043.2$ & 102.7 & 0.0 \\
\hline Somerset & $\mathrm{BR}$ & $5,446.4$ & 97.3 & 0.0 \\
\hline Gosnold & DU & $8,288.0$ & 89.7 & 0.0 \\
\hline $\mathrm{Hu} 11$ & PL & $1,619.2$ & 89.6 & 0.0 \\
\hline Oak Bluffs & DU & $4,640.0$ & 89.3 & 2.7 \\
\hline Hi ngham & PL & $14,457.6$ & 89.2 & 3.7 \\
\hline Rehoboth & $\mathrm{BR}$ & $30,374.4$ & 88.5 & 0.0 \\
\hline Kingston & PL & $12,179.2$ & 83.8 & 0.0 \\
\hline Tisbury & DU & $4,825.6$ & 53.6 & 0.0 \\
\hline Seekonk & $B R$ & $11,955.2$ & 52.8 & 0.0 \\
\hline Rockport & ES & $4,531.2$ & 48.2 & 0.0 \\
\hline Danvers & ES & $8,857.6$ & 34.9 & 0.0 \\
\hline Nahant & ES & 678.4 & 32.8 & 0.0 \\
\hline Salem & ES & $5,235.2$ & 30.8 & 0.0 \\
\hline Acushnet & $\mathrm{BR}$ & $12,038.4$ & 30.4 & 0.0 \\
\hline Beverly & $\mathrm{ES}$ & $9,830.4$ & 29.5 & 0.0 \\
\hline Gay Head & DU & $4,057.6$ & 29.0 & 6.6 \\
\hline West Tisbury & $\mathrm{DU}$ & $17,075.2$ & 20.9 & 0.0 \\
\hline Manchester & ES & $4,940.8$ & 19.1 & 0.0 \\
\hline Lynn & ES & $7,174.4$ & 17.6 & 0.0 \\
\hline Hanover & PL & $10,003.2$ & 16.2 & 0.0 \\
\hline Peabody & ES & $10,758.4$ & 9.0 & 0.0 \\
\hline Amesbury & ES & $8,838.4$ & 8.0 & 0.0 \\
\hline Braintree & NO & $9,222.4$ & 6.0 & 0.0 \\
\hline Marblehead & ES & $2,828.8$ & 6.0 & 0.0 \\
\hline Fall River & $B R$ & $24,371.2$ & 3.9 & 0.0 \\
\hline Chelsea & SU & $1,388.8$ & 2.0 & 0.0 \\
\hline Everett & MI & $2,400.0$ & 0.0 & 0.0 \\
\hline New Bedford & $\mathrm{BR}$ & $12,691.2$ & 0.0 & 0.0 \\
\hline Swamp scot $t$ & $\mathrm{ES}$ & $1,984.0$ & 0.0 & 0.0 \\
\hline State Totals & & $79,981.0$ & $48,104.6$ & 54.5 \\
\hline
\end{tabular}


Table 4

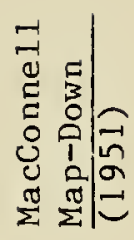

$m$
$\stackrel{m}{\sim}$
nn

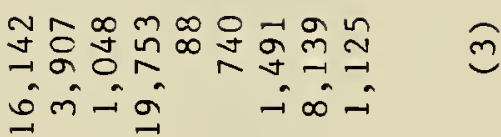

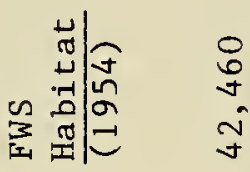

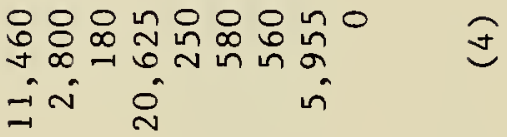

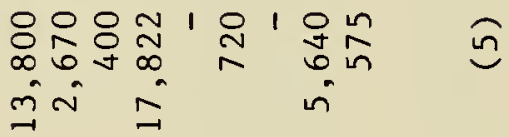

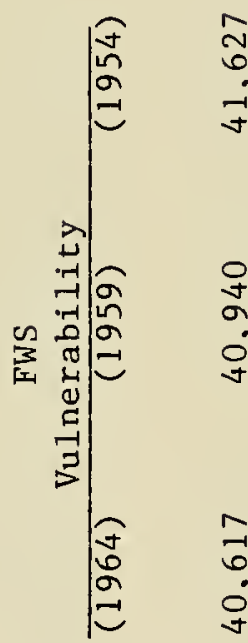

సै

ơ

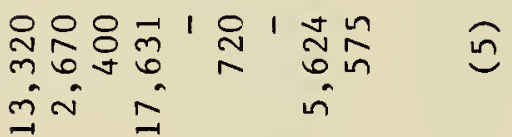

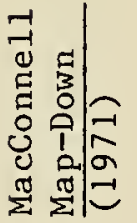

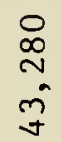

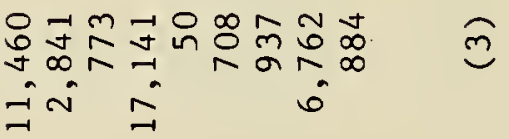

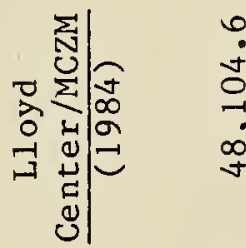

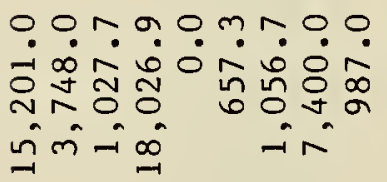

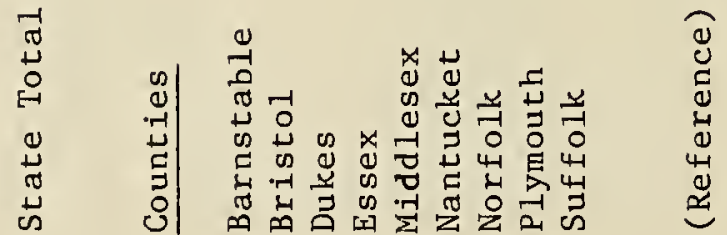




\section{TIDAL FLATS}

\section{$\underline{\text { Int roduction }}$}

Tidal flats, as defined by the coastal regulations to the Massachusetts Wetlands Protection Act, are those nearly level parts of coastal beaches which usually extend from the mean low water line landward to the more steeply sloping face of the beach, or which may be separated from the beach by an area of deeper water. They are exposed at low tide and may or may not be connected to the rest of a coastal beach. Tidal flats are commonly found both along shorelines exposed to the open ocean or within estuaries and the material making up the flat will generally reflect these differences in location; finer sediments are generally more common on protected flats and larger, sandy material more common in exposed areas. The values of tidal flats are often underestimated in the public eye. Most people recognize them as habitat for shellfish, however, they also play an important role in lessening storm waves and providing sediment to down-current beaches. The critical relationships with saltmarshes in the flow of nutrients within the estaurine or marine ecosystem are presently becoming better understood (6). For further information on the nature and values of tidal flats, the reader is referred to The Ecology of New England Tidal F1ats: a community profile (7).

\section{Study Methodology}

The methodology to determine tidal flat locations and areas was the same as that described above for salt marshes. Both estuarine and marine tidal flats (E2FL and M2FL respectively in the NWI indicator codes) were identified and planimetered on black line copies of the National Wetlands Inventory maps. Again, one half acre was the minimum threshold for resource area size used in developing the NWI maps.

To our knowledge, this is the first systematic survey of tidal flats within the Commonwealth.

Table 5 lists acreages by town within counties. Table 6 provides the acreage of tidal flats by community in descending, sequential order. In each case, totals are provided for both marine (open ocean) and estuarine (within bays, river mouths, salt ponds, etc.) flats and combined as tidal flats.

\section{Discussion}

The north side of Cape Cod (Barnstable County) has extensive marine flats as may be seen in the figures of Eastham, Brewster, Wellfleet and Yarmouth. Estuarine flats are concentrated in such areas as Duxbury Bay, Pleasant Bay (Chatham and orleans), Barnstable Harbor, Plymouth Bay, and the Westport River. 
Table 5

TIDAL FLAT ACREAGE WITHIN COUNTIES

Town

\section{Barnstable County}

Eastham
Barnstable
Orleans
Brewster
Chatham
Wellfleet
Yarmouth
Dennis
Falmouth
Provincetown
Bourne
Truro
Mashpee
Sandwich
Harwich

Town Area in Acres

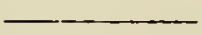

$$
9,344.0
$$

$40,153.6$

$9,081.6$

$16,102.4$

$10,438.4$

$13,324.8$

$16,339.2$

$14,016.0$

$29,260.8$

$5,600.0$

$26,585.6$

$13,824.0$

$16,614.4$

$27,916.8$

Totals $\frac{14,342.4}{262,944.0}$

Bristol County

Westport
Fairhaven
Dartmouth
New Bedford
Berkley
Acushnet
Swansea
Dighton
Somerset
Fall River
Freetown
Rehoboth
Seekonk

\begin{tabular}{r}
$35,353.6$ \\
$7,936.0$ \\
$39,558.4$ \\
$12,691.2$ \\
$10,496.0$ \\
$12,038.4$ \\
$14,585.6$ \\
$14,304.0$ \\
$5,446.4$ \\
$24,371.2$ \\
$23,174.4$ \\
$30,374.4$ \\
$11,955.2$ \\
\hline \\
Totals $\quad 242,284.8$
\end{tabular}

Dukes County

Edgartown

Tisbury

Chilmark

Gay Head

Oak Bluffs

Gosnold

West Tisbury

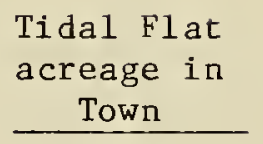

Marine

Flat

Acreage

\section{$2,817.9$}

$2,646.4$

$2,441.1$

$2,367.2$

$2,231.9$

$1,893.7$

$1,542.1$

$1,002.1$

307.5

205.4

201.8

92.5

51.6

7.4

0.0

$\frac{0.0}{17,808.6}$

$2,032.8$

582.1

281.7

107.7

53.6

49.8

11.4

7.0

4.4

0.0

0.0

0.0

$\frac{0.0}{3,130.5}$

Totals $\frac{17,075.2}{71,788.8}$
786.9

221.1

119.5

47.8

37.9

34.1

$$
\begin{array}{r}
18,720.0 \\
4,825.6 \\
14,182.4 \\
4,057.6 \\
4,640.0 \\
8,288.0
\end{array}
$$$$
11.4
$$$$
1,258.7
$$

Estuarine

Flat

Acreage

542.5

$1,957.2$

$2,027.6$

14.9

$1,133.9$

786.5

476.8

122.0

180.2

162.4

166.0

92.5

51.6

7.4

0.0

0.0

0.0 
Essex County

Ipswich

Gloucester

Newburyport

Essex

Newbury

Salem

Beverly

Rowley

Manchester

Salisbury

Marblehead

Danvers

Saugus

Rockport

Lynn

Amesbury

Nahant

Peabody

Swamp scott

$$
\begin{array}{r}
21,344.0 \\
16,928.0 \\
5,702.4 \\
9,203.4 \\
15,577.6 \\
5,235.2 \\
9,830.4 \\
12,179.2 \\
4,940.8 \\
10,323.2 \\
2,828.8 \\
8,857.6 \\
7,411.2 \\
4,531.2 \\
7,174.4 \\
8,838.4 \\
678.4 \\
10,758.4
\end{array}
$$$$
\text { Totals } \frac{1,984.0}{164,326.6}
$$

Middlesex County

$$
\text { Everett }
$$

Nantucket County

Nantucket

Norfolk County

Quincy
Weymouth
Cohasset
Braintree
Milton

Plymouth County

Duxbury
Plymouth
Kingston
Scituate
Hingham
Hull
Mattapoiset
Wareham
Marshfield
Marion
Hanover
Norwel1
Pembroke

$32,217.6$

$10,649.6$

$11,340.8$

$6,438.4$

$9,222.4$

Totals $\frac{8,448.0}{46,099.2}$

$10,003.2$
$1,539.9$

$1,412.9$ 690.7

512.5

431.6

317.0

303.9

211.2

127.6

115.5

112.2

96.5

92.2

74.7

49.3

0.0

0.0

0.0

$\frac{0.0}{6,087.7}$

0.0

0.0

0.0

136.9

0.0

136.9
$1,459.6$
549.7
287.6
37.7
$\frac{0.0}{2,334.6}$

0.0

0.0

0.0

0.0

$\frac{0.0}{0.0}$

$1,459.6$

549.7

287.6

37.7

$\frac{0.0}{2,334.6}$

Totals $\frac{14,886.4}{222,387.2}$
0.0

20.3

0.0

0.0

0.0

0.0

439.5

256.3

0.0

46.7

0.0

0.0

$\frac{0.0}{762.8}$
$3,436.8$

$2,109.5$

905.0

621.8

614.5

511.0

453.6

451.2

172.9

93.6

0.0

0.0

$3,436.8$

$2,089.2$

905.0

621.8

614.5

511.0

14.1

194.9

172.9

46.9

0.0

0.0

$\frac{0.0}{9,369.9}$ $\frac{0.0}{8,607.1}$

Suffolk County

Boston

Winthrop

Revere

Chelsea

\begin{tabular}{rrr} 
& $29,056.0$ & 878.5 \\
& $1,043.2$ & 295.8 \\
& $4,044.8$ & 183.5 \\
Totals & $1,388.8$ & 29.5 \\
\cline { 2 - 3 } & $35,532.8$ & $1,387.3$
\end{tabular}

878.5

0.0

0.0

129.1

$\frac{0.0}{129.1}$
878.5

295.8

54.4

29.5 
Table 6

TIDAL FLAT ACREAGE BY TOWN (arranged in descending order)

\begin{tabular}{|c|c|c|c|c|c|}
\hline Town & County & Town Area & Tidal Flats & $\begin{array}{l}\text { Marine } \\
\text { Flats } \\
\end{array}$ & $\begin{array}{c}\text { Estuarine } \\
\text { Flats } \\
\end{array}$ \\
\hline Duxbury & PL & $15,686.4$ & $3,436.8$ & 0.0 & $3,436.8$ \\
\hline Eastham & $\mathrm{BA}$ & $9,344.0$ & $2,817.9$ & $2,275.4$ & 542.5 \\
\hline Barnstable & $\mathrm{BA}$ & $40,153.6$ & $2,646.4$ & 689.2 & $1,957.2$ \\
\hline Orleans & $\mathrm{BA}$ & $9,081.6$ & $2,441.1$ & 413.5 & $2,027.6$ \\
\hline Brewster & $\mathrm{BA}$ & $16,102.4$ & $2,367.2$ & $2,352.3$ & 14.9 \\
\hline Chatham & $\mathrm{BA}$ & $10,438.4$ & $2,231.9$ & $1,098.0$ & $1,133.9$ \\
\hline Plymouth & PL & $66,048.0$ & $2,109.5$ & 20.3 & $2,089.2$ \\
\hline Westport & $\mathrm{BR}$ & $35,353.6$ & $2,032.8$ & 0.0 & $2,032.8$ \\
\hline Wellfleet & $\mathrm{BA}$ & $13,324.8$ & $1,893.7$ & $1,107.2$ & 786.5 \\
\hline Yarmouth & BA & $16,339.2$ & $1,542.1$ & $1,065.3$ & 476.8 \\
\hline Ipswich & ES & $21,344.0$ & $1,539.9$ & 516.8 & $1,023.1$ \\
\hline Quincy & NO & $10,649.6$ & $1,459.6$ & 0.0 & $1,459.6$ \\
\hline Gloucester & ES & $16,928.0$ & $1,412.9$ & 510.0 & 902.9 \\
\hline Dennis & BA & $14,016.0$ & $1,002.1$ & 880.1 & 122.0 \\
\hline Kingston & PL & $12,179.2$ & 905.0 & 0.0 & 905.0 \\
\hline Boston & SU & $29,056.0$ & 878.5 & 0.0 & 878.5 \\
\hline Edgartown & DU & $18,720.0$ & 786.9 & 154.1 & 632.8 \\
\hline Newburyport & ES & $5,702.4$ & 690.7 & 0.0 & 690.7 \\
\hline Scituate & PL & $10,924.8$ & 621.8 & 0.0 & 621.8 \\
\hline Hingham & PL & $14,457.6$ & 614.5 & 0.0 & 614.5 \\
\hline Fairhaven & $\mathrm{BR}$ & $7,936.0$ & 582.1 & 442.0 & 140.1 \\
\hline Weymouth & NO & $11,340.8$ & 549.7 & 0.0 & 549.7 \\
\hline Essex & ES & $9,203.4$ & 512.5 & 0.0 & 512.5 \\
\hline Hul1 & PL & $1,619.2$ & 511.0 & 0.0 & 511.0 \\
\hline Mattapoiset & PL & $11,187.2$ & 453.6 & 439.5 & 14.1 \\
\hline Wareham & PL & $24,339.2$ & 451.2 & 256.3 & 194.9 \\
\hline Newbury & ES & $15,577.6$ & 431.6 & 127.7 & 303.9 \\
\hline Salem & ES & $5,235.2$ & 317.0 & 196.0 & 121.0 \\
\hline Falmouth & BA & $29,260.8$ & 307.5 & 127.3 & 180.2 \\
\hline Beverly & ES & $9,830.4$ & 303.9 & 198.5 & 105.4 \\
\hline Winthrop & $\mathrm{SU}$ & $1,043.2$ & 295.8 & 0.0 & 295.8 \\
\hline Cohasset & NO & $6,438.4$ & 287.6 & 0.0 & 287.6 \\
\hline Dartmouth & $\mathrm{BR}$ & $39,558.4$ & 281.7 & 14.7 & 267.0 \\
\hline Tisbury & $\mathrm{DU}$ & $4,825.6$ & 221.1 & 0.0 & 221.1 \\
\hline Rowley & $\mathrm{ES}$ & $12,179.2$ & 211.2 & 12.6 & 198.6 \\
\hline Provincetown & $\mathrm{BA}$ & $5,600.0$ & 205.4 & 43.0 & 162.4 \\
\hline Bourne & $\mathrm{BA}$ & $26,585.6$ & 201.8 & 35.8 & 166.0 \\
\hline Revere & SU & $4,044.8$ & 183.5 & 129.1 & 54.4 \\
\hline Marshfield & PL & $18,252.8$ & 172.9 & 0.0 & 172.9 \\
\hline Nantucket & NA & $32,217.6$ & 136.9 & 0.0 & 136.9 \\
\hline
\end{tabular}


TIDAL FLAT ACREAGE BY TOWN (continued)

\begin{tabular}{|c|c|c|c|c|c|}
\hline Town & County & Town Area & Tidal Flats & $\begin{array}{l}\text { Marine } \\
\text { Flats } \\
\end{array}$ & $\begin{array}{c}\text { Estuarine } \\
\text { Flats }\end{array}$ \\
\hline Manchester & ES & $4,940.8$ & 127.6 & 111.4 & 16.2 \\
\hline Chilmark & $\mathrm{DU}$ & $14,182.4$ & 119.5 & 6.0 & 113.5 \\
\hline Salisbury & ES & $10,323.2$ & 115.5 & 115.5 & 0.0 \\
\hline Marblehead & ES & $2,828.8$ & 112.2 & 112.2 & 0.0 \\
\hline New Bedford & $\mathrm{BR}$ & $12,691.2$ & 107.7 & 0.0 & 107.7 \\
\hline Danvers & ES & $8,857.6$ & 96.5 & 0.0 & 96.5 \\
\hline Marion & PL & $9,152.0$ & 93.6 & 46.7 & 46.9 \\
\hline Truro & BA & $13,824.0$ & 92.5 & 0.0 & 92.5 \\
\hline Saugus & ES & $7,411.2$ & 92.2 & 0.0 & 92.2 \\
\hline Rockport & ES & $4,531.2$ & 74.7 & 74.7 & 0.0 \\
\hline Berkley & $\mathrm{BR}$ & $10,496.0$ & 53.6 & 0.0 & 53.6 \\
\hline Mashpee & BA & $16,614.4$ & 51.6 & 0.0 & 51.6 \\
\hline Acushnet & BR & $12,038.4$ & 49.8 & 0.0 & 49.8 \\
\hline Lynn & $\mathrm{ES}$ & $7,174.4$ & 49.3 & 0.0 & 49.3 \\
\hline Gay Head & DU & $4,057.6$ & 47.8 & 0.0 & 47.8 \\
\hline Dak Bluffs & $\mathrm{DU}$ & $4,640.0$ & 37.9 & 0.0 & 37.9 \\
\hline Braintree & NO & $9,222.4$ & 37.7 & 0.0 & 37.7 \\
\hline Gosnold & DU & $8,288.0$ & 34.1 & 25.4 & 8.7 \\
\hline Chelsea & SU & $1,388.8$ & 29.5 & 0.0 & 29.5 \\
\hline Swansea & $\mathrm{BR}$ & $14,585.6$ & 11.4 & 0.0 & 11.4 \\
\hline West Tisbury & $\mathrm{DU}$ & $17,075.2$ & 11.4 & 0.0 & 11.4 \\
\hline Sandwich & $\mathrm{BA}$ & $27,916.8$ & 7.4 & 0.0 & 7.4 \\
\hline Dighton & $\mathrm{BR}$ & $14,304.0$ & 7.0 & 0.0 & 7.0 \\
\hline Somerset & $\mathrm{BR}$ & $5,446.4$ & 4.4 & 0.0 & 4.4 \\
\hline Amesbury & ES & $8,838.4$ & 0.0 & 0.0 & 0.0 \\
\hline Everett & MI & $2,400.0$ & 0.0 & 0.0 & 0.0 \\
\hline Fall River & $\mathrm{BR}$ & $24,371.2$ & 0.0 & 0.0 & 0.0 \\
\hline Freetown & BR & $23,174.4$ & 0.0 & 0.0 & 0.0 \\
\hline Hanover & PL & $10,003.2$ & 0.0 & 0.0 & 0.0 \\
\hline Harwich & $\mathrm{BA}$ & $14,342.4$ & 0.0 & 0.0 & 0.0 \\
\hline Milton & NO & $8,448.0$ & 0.0 & 0.0 & 0.0 \\
\hline Nahant & ES & 678.4 & 0.0 & 0.0 & 0.0 \\
\hline Norwe11 & PL & $13,651.2$ & 0.0 & 0.0 & 0.0 \\
\hline Pe abody & ES & $10,758.4$ & 0.0 & 0.0 & 0.0 \\
\hline Pembroke & PL & $14,886.4$ & 0.0 & 0.0 & 0.0 \\
\hline Rehoboth & $B R$ & $30,374.4$ & 0.0 & 0.0 & 0.0 \\
\hline Seekonk & $\mathrm{BR}$ & $11,955.2$ & 0.0 & 0.0 & 0.0 \\
\hline Swamps cott & $\mathrm{ES}$ & $1,984.0$ & 0.0 & 0.0 & 0.0 \\
\hline State Totals & & $1,079,981.0$ & $41,514.2$ & $13,596.6$ & $27,917.6$ \\
\hline
\end{tabular}


BARRIER BEACHES

\section{Introduction}

Barrier beaches are geologic landforms defined in the regulations to the Massachusetts Wetlands Protection Act as narrow, low-lying strips of land generally consisting of coastal beaches and coastal dunes extending roughly parallel to the trend of the coast. They are separated from the mainland by a relatively narrow body of fresh, brackish, or saline water or by a salt marsh system. A barrier beach may be joined to the mainland at one or both ends.

Storm damage prevention and flood control are two of the most obvious values of these resource areas. The sands of a barrier beach can absorb the force of storm waves, and the reshaping of beaches and dunes by waves provides material to beaches down-current and eases the effects of erosion. Barrier beaches are also often important recreational areas and provide nesting and resting sites for many species of shore and migratory birds.

For further information about barrier beaches, their resources and management, see the Barrier Beach Management Sourcebook (8) and Massachusetts Barrier Beaches (9).

\section{Study Methodology}

The acreage of barrier beach units was developed from an inventory prepared for the Massachusetts Coastal Zone Management (MCZM) program by the Provincetown Center for Coastal Studies. Delineation of barrier units was made on US Geological Survey $71 / 2$ minute quadrangle topographic sheets. Original versions of these maps are on file in the MCZM office. Reduced size versions as well as identification criteria and study methodology information are available in Massachusetts Barrier Beaches (9) or through MCZM.

As with the salt marshes and tidal flats described above, each defined area was traced using the Numonics digital read-out planimeter at the US Fish and Wildlife Service' Region V office. Numbers of barrier units and their acreage were recorded by town and county. County and state acreages are presented in Table 1. Numbers of barriers and acreages listed by town within county sequence are found in Table 7 . Table 8 lists barrier beach acreage by town.

\section{Discussion}

A size distribution of the Commonwealth's barriers was prepared and is presented in Table 9. For the purposes of this analysis, the acreage of the entire geologic landform was used, despite any political boundaries. The barrier beach inventory is based on community units and a large barrier such as Plum Island may have its acreage divided among several towns. The distribution analysis indicates that most of the 661 barriers of Massachusetts are relatively small. 
More than half are less than 2.5 acres and more than three-quarters are less than 10 acres. Conversely, the 30 largest barriers make up three-quarters of the total barrier beach acreage in the state. These 30 barrier landforms are listed in Table 10 .

Interestingly, the Town of Gosnold (the Elizabeth Islands) has the largest number of barriers, and, in fact, has more barrier beaches (77) than year-round residents (63) according to the 1980 census. 
Table 7

NUMBERS OF BARRIER BEACH SEGMENTS AND ACREAGE WITHIN COUNTIES

Town

\section{Barnstable County}

Falmouth
Barnstable
Bourne
Wellfleet
Chatham
Orleans
Yarmouth
Dennis
Eastham
Harwich
Truro
Mashpee
Sandwich
Brewster
Provincetown

Bristol County

Fairhaven

Dartmouth

Westport

Swansea

Somerset

Acushnet

Berkley

Dighton

Fall River

Freetown

New Bedford

Rehoboth

Seekonk

Dukes County

Gosnold

Edgartown

Chilmark

West Tisbury

Oak Bluffs

Tisbury

Gay Head
Town Area

in Acres \begin{tabular}{c} 
Barrier Beach \\
Acreage in \\
Town \\
\hline
\end{tabular}

Barrier Beach

Segments in Town

\begin{tabular}{rr}
278.4 & 44 \\
$1,700.7$ & 29 \\
75.0 & 29 \\
164.4 & 22 \\
$3,108.1$ & 20 \\
532.1 & 17 \\
212.5 & 13 \\
232.2 & 12 \\
224.7 & 7 \\
27.1 & 7 \\
$1,001.1$ & 7 \\
128.5 & 6 \\
259.5 & 5 \\
53.0 & 4 \\
725.8 & 2 \\
\hline $8,723.1$ & 224
\end{tabular}

$29,260.8$

$40,153.6$

$26,585.6$

$13,324.8$

$10,438.4$

$9,081.6$

$16,339.2$

$14,016.0$

$9,344.0$

$14,342.4$

$13,824.0$

$16,614.4$

$27,916.8$

$16,102.4$

Totals $\frac{5,600.0}{262,944.0}$

Town

4

9

22

20

17

12

7

7

7

5

4

224

\section{3}

154.4

729.4

33.5

4.5

0.0

0.0

0.0

0.0

0.0

0.0

0.0

0.0

$1,008.1$

23

13

6

2

1

0

0

0

0

$23,174.4$

$12,691.2$

$30,374.4$

Totals $\frac{11,955.2}{242,284.8}$

$$
\begin{array}{r}
8,288.0 \\
18,720.0 \\
14,182.4 \\
17,075.2 \\
4,640.0 \\
4,825.6
\end{array}
$$$$
\text { Totals } \frac{4,057.6}{71,788.8}
$$

186.3

77

40

19

297.0

82.5

115.4

17

13

88.1

13

$2, \frac{403.8}{135.7}$ 
Essex County

Gloucester
Ipswich
Rockport
Manchester
Nahant
Beverly
Marblehead
Newbury
Newburyport
Rowley
Salisbury
Swampscott
Amesbury
Danvers
Essex
Lynn
Peabody
Salem
Saugus

\begin{tabular}{rr}
$16,928.0$ \\
$21,344.0$ \\
$4,531.2$ \\
$4,940.8$ \\
678.4 \\
$9,830.4$ \\
$2,828.8$ \\
$15,577.6$ \\
$5,702.4$ \\
$12,179.2$ \\
$10,323.2$ \\
$1,984.0$ \\
$8,838.4$ \\
$8,857.6$ \\
$9,203.4$ \\
$7,174.4$ \\
$10,758.4$ \\
$5,235.2$ \\
$7,411.2$ \\
\hline $164,326.6$
\end{tabular}

\begin{tabular}{rr}
171.6 & 9 \\
$1,333.3$ & 6 \\
35.6 & 5 \\
10.7 & 4 \\
80.0 & 3 \\
1.5 & 1 \\
14.6 & 1 \\
606.6 & 1 \\
166.1 & 1 \\
186.0 & 1 \\
345.3 & 1 \\
4.1 & 1 \\
0.0 & 0 \\
0.0 & 0 \\
0.0 & 0 \\
0.0 & 0 \\
0.0 & 0 \\
0.0 & 0 \\
0.0 & 0 \\
\hline $2,955.4$ & 34
\end{tabular}

Middlesex County Everett

$$
2,400.0
$$

0.0

0

Nantucket County Nantucket

$$
32,217.6
$$

$1,841.7$

Norfolk County
Quincy
Cohasset
Weymouth
Braintree
Milton

Plymouth County

Wareham
Mattapoiset

Marion

Plymouth

Scituate

Hull

Marshfield

Hingham

Duxbury

Hanover

Kingston

Norwel1

Pembroke

Suffolk County

Boston

Revere

Winthrop

Chelsea

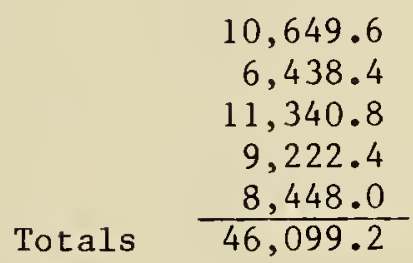

$$
\begin{array}{r}
71.4 \\
20.1 \\
4.2 \\
0.0 \\
0.0 \\
\hline 95.7
\end{array}
$$$$
\begin{array}{r}
6 \\
3 \\
1 \\
0 \\
0 \\
\hline 10
\end{array}
$$

\section{7}

83.5

36.9

348.0

323.1

599.0

232.1

4.8

247.7

0.0

0.0

0.0

$13,651.2$

\section{6}

26

14

12

11

9

6

3

1

0

0

0

$\frac{0}{118}$
Totals $\frac{14,886.4}{222,387.2}$

$\frac{0.0}{1,933.8}$

28.4

151.2

14.9

$4,044.8$

$1,043.2$

Totals $\frac{1,388.8}{35,532.8}$ 
Table 8

BARRIER BEACH ACREAGE BY TOWN (arranged in descending order)

\begin{tabular}{|c|c|c|c|c|}
\hline Town & County & $\begin{array}{l}\text { Town Area } \\
\text { in acres }\end{array}$ & $\begin{array}{l}\text { Barrier Beach } \\
\text { Acreage within } \\
\text { Town } \\
\end{array}$ & $\begin{array}{l}\text { Number of } \\
\text { barriers in } \\
\text { Town } \\
\end{array}$ \\
\hline Chatham & $\mathrm{BA}$ & $10,438.4$ & $3,108.1$ & 20 \\
\hline Nantucket & NA & $32,217.6$ & $1,841.7$ & 56 \\
\hline Barnstable & $\mathrm{BA}$ & $40,153.6$ & $1,700.7$ & 29 \\
\hline Ipswich & ES & $21,344.0$ & $1,333.3$ & 6 \\
\hline Truro & $\mathrm{BA}$ & $13,824.0$ & $1,001.1$ & 7 \\
\hline Edgartown & DU & $18,720.0$ & 962.6 & 40 \\
\hline Westport & $B R$ & $35,353.6$ & 729.4 & 6 \\
\hline Provincetown & BA & $5,600.0$ & 725.8 & 2 \\
\hline Newbury & ES & $15,577.6$ & 606.6 & 1 \\
\hline Hul1 & $\mathrm{PL}$ & $1,619.2$ & 599.0 & 9 \\
\hline Orleans & BA & $9,081.6$ & 532.1 & 17 \\
\hline Gay Head & $\mathrm{DU}$ & $4,057.6$ & 403.8 & 3 \\
\hline Plymouth & PL & $66,048.0$ & 348.0 & 12 \\
\hline Salisbury & $\mathrm{ES}$ & $10,323.2$ & 345.3 & 1 \\
\hline Scituate & PL & $10,924.8$ & 323.1 & 11 \\
\hline Chilmark & DU & $14,182.4$ & 297.0 & 19 \\
\hline Falmouth & $\mathrm{BA}$ & $29,260.8$ & 278.4 & 44 \\
\hline Sandwich & $\mathrm{BA}$ & $27,916.8$ & 259.5 & 5 \\
\hline Duxbury & PL & $15,686.4$ & 247.7 & 1 \\
\hline Dennis & $\mathrm{BA}$ & $14,016.0$ & 232.2 & 12 \\
\hline Marshfield & PL & $18,252.8$ & 232.1 & 6 \\
\hline Eastham & $\mathrm{BA}$ & $9,344.0$ & 224.7 & 7 \\
\hline Yarmouth & BA & $16,339.2$ & 212.5 & 13 \\
\hline Gosnold & $\mathrm{DU}$ & $8,288.0$ & 186.3 & 77 \\
\hline Rowley & ES & $12,179.2$ & 186.0 & 1 \\
\hline Gloucester & ES & $16,928.0$ & 171.6 & 9 \\
\hline Newburyport & ES & $5,702.4$ & 166.1 & 1 \\
\hline Wellf leet & $\mathrm{BA}$ & $13,324.8$ & 164.4 & 22 \\
\hline Dartmouth & $\mathrm{BR}$ & $39,558.4$ & 154.4 & 13 \\
\hline Revere & SU & $4,044.8$ & 151.2 & 2 \\
\hline Mashpee & BA & $16,614.4$ & 128.5 & 6 \\
\hline Oak Bluffs & DU & $4,640.0$ & 115.4 & 13 \\
\hline Tisbury & DU & $4,825.6$ & 88.1 & 13 \\
\hline Fairhaven & $\mathrm{BR}$ & $7,936.0$ & 86.3 & 23 \\
\hline Mattapoiset & PL & $11,187.2$ & 83.5 & 26 \\
\hline West Tisbury & DU & $17,075.2$ & 82.5 & 17 \\
\hline Nahant & ES & 678.4 & 80.0 & 3 \\
\hline Bourne & BA & $26,585.6$ & 75.0 & 29 \\
\hline Quincy & NO & $10,649.6$ & 71.4 & 6 \\
\hline Wareham & PL & $24,339.2$ & 58.7 & 36 \\
\hline
\end{tabular}


BARRIER BEACH ACREAGE BY TOWN (continued)

\begin{tabular}{|c|c|c|c|c|}
\hline Town & County & $\begin{array}{l}\text { Town Area } \\
\text { in acres }\end{array}$ & $\begin{array}{l}\text { Barrier Beach } \\
\text { Acreage within } \\
\text { Town } \\
\end{array}$ & $\begin{array}{l}\text { Number of } \\
\text { barriers in } \\
\text { Town } \\
\end{array}$ \\
\hline Brewster & BA & $16,102.4$ & 53.0 & 4 \\
\hline Marion & PL & $9,152.0$ & 36.9 & 14 \\
\hline Rockport & ES & $4,531.2$ & 35.6 & 5 \\
\hline Swansea & $\mathrm{BR}$ & $14,585.6$ & 33.5 & 2 \\
\hline Boston & $\mathrm{SU}$ & $29,056.0$ & 28.4 & 11 \\
\hline Harwich & BA & $14,342.4$ & 27.1 & 7 \\
\hline Cohasset & NO & $6,438.4$ & 20.1 & 3 \\
\hline Winthrop & SU & $1,043.2$ & 14.9 & 2 \\
\hline Marblehead & ES & $2,828.8$ & 14.6 & 1 \\
\hline Manchester & ES & $4,940.8$ & 10.7 & 4 \\
\hline Hingham & PL & $14,457.6$ & 4.8 & 3 \\
\hline Somerset & $B R$ & $5,446.4$ & 4.5 & 1 \\
\hline Weymouth & NO & $11,340.8$ & 4.2 & 1 \\
\hline Swamp scot $t$ & $\mathrm{ES}$ & $1,984.0$ & 4.1 & 1 \\
\hline Beverly & ES & $9,830.4$ & 1.5 & 1 \\
\hline Acushnet & $\mathrm{BR}$ & $12,038.4$ & 0.0 & 0 \\
\hline Amesbury & ES & $8,838.4$ & 0.0 & 0 \\
\hline Berkley & $\mathrm{BR}$ & $10,496.0$ & 0.0 & 0 \\
\hline Braintree & NO & $9,222.4$ & 0.0 & 0 \\
\hline Chelsea & $\mathrm{SU}$ & $1,388.8$ & 0.0 & 0 \\
\hline Danvers & ES & $8,857.6$ & 0.0 & 0 \\
\hline Dighton & $B R$ & $14,304.0$ & 0.0 & 0 \\
\hline Essex & ES & $9,203.4$ & 0.0 & 0 \\
\hline Everett & MI & $2,400.0$ & 0.0 & 0 \\
\hline Fal1 River & $\mathrm{BR}$ & $24,371.2$ & 0.0 & 0 \\
\hline Freetown & $\mathrm{BR}$ & $23,174.4$ & 0.0 & 0 \\
\hline Hanover & PL & $10,003.2$ & 0.0 & 0 \\
\hline Kingston & PL & $12,179.2$ & 0.0 & 0 \\
\hline Lynn & ES & $7,174.4$ & 0.0 & 0 \\
\hline Milton & NO & $8,448.0$ & 0.0 & 0 \\
\hline New Bedford & BR & $12,691.2$ & 0.0 & 0 \\
\hline Norwe11 & PL & $13,651.2$ & 0.0 & 0 \\
\hline Peabody & ES & $10,758.4$ & 0.0 & 0 \\
\hline Pembroke & PL & $14,886.4$ & 0.0 & 0 \\
\hline Rehoboth & $\mathrm{BR}$ & $30,374.4$ & 0.0 & 0 \\
\hline Salem & $\mathrm{ES}$ & $5,235.2$ & 0.0 & 0 \\
\hline Saugus & ES & $7,411.2$ & 0.0 & 0 \\
\hline Seekonk & $\mathrm{BR}$ & $11,955.2$ & 0.0 & 0 \\
\hline
\end{tabular}

State Totals 
Table 9

SIZE DISTRIBUTION OF MASSACHUSETTS BARRIER BEACHES

\begin{tabular}{|c|c|c|c|c|c|c|c|c|}
\hline Range & of & Acreage & wit & $\begin{array}{c}\text { range } \alpha \\
\text { Total }\end{array}$ & & $\begin{array}{r}\text { Cumu 1.a } \\
\%\end{array}$ & $\begin{array}{l}\text { tot } \\
\text { Tota }\end{array}$ & $\begin{array}{l}\text { tal } \\
\text { a }\end{array}$ \\
\hline 0 & - & .4 & 47 & $7 \%$ & & & & \\
\hline .5 & - & .9 & 94 & $14 \%$ & & 141 & 21 & \\
\hline 1.0 & - & 1.4 & 88 & $13 \%$ & & 229 & 35 & \\
\hline 1.5 & - & 1.9 & 59 & $9 \%$ & & 288 & 44 & $\%$ \\
\hline 2.0 & - & 2.9 & 69 & $10 \%$ & & 357 & 54 & \\
\hline 3.0 & - & 4.9 & 70 & $11 \%$ & & 427 & 65 & $\%$ \\
\hline 5.0 & - & 9.9 & 87 & $13 \%$ & & 514 & 78 & $\%$ \\
\hline 10.0 & - & 49.9 & 99 & $15 \%$ & & 613 & 93 & \\
\hline 50.0 & - & 99.9 & 18 & $3 \%$ & & 631 & 95 & $\%$ \\
\hline 100.0 & -5 & 999.9 & 25 & $4 \%$ & & 656 & 99 & $\%$ \\
\hline ov & ver & 1000 & 5 & less & than $1 \%$ & 661 & 100 & $\%$ \\
\hline
\end{tabular}

The 5 largest barriers ( $1 \%$ of total) make up $38 \%$ of total acres. The 30 largest barriers ( $5 \%$ of tota1) make up $75 \%$ of total acres.

Al1 areas given in acres. 
Table 10

THE 30 LARGEST BARRIER BEACH LANDFORMS IN MASSACHUSETTS

$\begin{array}{ll}\text { Acreage } & \text { Barrier Name } \\ 2126.8 & \text { Monomoy Island } \\ 1381.7 & \text { Sandy Neck } \\ 1255.0 & \text { Nauset Beach } \\ 1241.4 & \text { Plum Island }\end{array}$

1119.6 Coatue Point and Beach

875.2 Castle Neck/Cranes Beach

789.0 Head of the Meadow Beach

651.7 Horseneck Beach

527.7 Nantasket Beach

442.7 Race Point/Hatches Harbor

345.3 Salisbury Beach

337.5 Lobsterville/West Payson Road

310.8 Duxbury Beach

301.2 Cape Poge

283.1 Wood End/Long Point

270.6 Hummarock/Rexhame

223.5 Norton Point

149.7 Revere Beach

138.7 Wauwinet

135.7 Coast Guard/Nauset Beach

134.6 Great Is land

133.5 Chapin Beach

132.2 Beach Point

129.3 Edgartown Great Pond

127.0 Squibnocket/Long Beach

124.1 P1ymouth Beach

122.2 East Sandwich/Springhill

121.2 Eel Point

116.4 Dead Neck

103.3 Ester Is land
$\operatorname{Town}(\mathrm{s})$

Chatham

Barnstable, Sandwich Chatham, Or leans

Ipswich, Newbury, Newburyport, Rowley

Nantucket

Ipswich, Gloucester

Truro

Westport

Hull

Provincetown

Salisbury

Gay Head

Duxbury, Marshfield,

Plymouth

Edgartown

Provincetown

Marshfield, Scituate

Edgartown

Revere

Nantucket

Eastham

Yarmouth

Dennis

Truro

Edgartown

Chilmark, Gay Head

Plymouth

Sandwich

Nantucket

Barnstable

Nant ucket
County

Barnstable

Barnstable

Barnstable

Essex

Nantucket

Essex

Barnstable

Bristol

Plymouth

Barnstable

Essex

Dukes

Plymouth

Dukes

Barnstable

Plymouth

Dukes

Suffolk

Nantucket

Barnstable

Barnstable

Barnstable

Barnstable

Dukes

Dukes

Plymouth

Barnstable

Nantucket

Barnstable

Nantucket 
REFERENCES

(1) Nixon, Scott $W$.

1982

The Ecology of New England High Salt Marshes: A community profile. US Fish and Wildiife Service, Of fice of Biological Services, Washington, D.C. FWS/OBS - 81/55 $70 \mathrm{pp}$.

(2) Cowardin, L.M., V. Carter, F.C. Golet, and E.T. LaRoe 1979

Classification of Wetlands and Deepwater Habitats of the United States.

US Fish and Wildlife Service, Of fice of Biological Services, Washington, D.C. FWS/OBS - 79/31 103 pp.

MacConnel1, William P.

1975

Remote Sensing 20 Years of Change in Massachusetts, 1951/52-

Massachusetts Agricultural Experiment Station, University of Massachusetts, Amherst, MA. Research Bulletin 非630 79 pp.

(4) US Fish and Wildlife Service

1954

Wetlands Inventory of Massachusetts

Office of River Basin Studies, US Fish and Wildlife Service, Boston, MA. 28 pp.

US Fish and Wildlife Service 1965

Supplementary Report (June 1965) on the Coastal Wetlands Inventory of Massachusetts.

US Fish and Wildlife Service, Bureau of Sport Fisheries and Wildlife, Region V, Boston, MA. 13 pp.

(6) Welsh, Barbara L. 1980

Comparitive Nutrient Dynamics of a Marsh-Mudflat Ecosystem. Estuarine and Coastal Marine Sciences (1980), 10, 143-164. 
$-25-$

(7)

(8)

(9)
Whitlatch, Robert B. 1982

The Ecology of New England Tidal Flats: A community profile. US Fish and Wildlife Service, Of ice of Biological Services, Washington, DC, FWS/OBS - 81/01 $125 \mathrm{pp}$.
Smith, Lester B., Jr.

1983

Barrier Beach Management Sourcebook.

Massachusetts Coastal Zone Management Program, Boston, MA. 48 pp.

Massachusetts Coastal Zone Management Program 1979

Massachusetts Barrier Beaches.

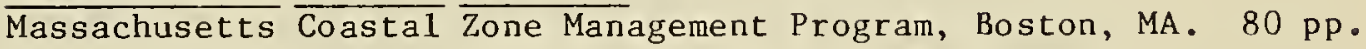




\section{APPENDIX}

\section{Classification Scheme for Salt Marshes and Tidal Flats}

The classification scheme used on the National Wetlands Inventory maps is that provided in Classification of Wetlands and Deepwater Habitats of the United States (2). This is based on a hierarchical model of System/ Sub-system Class / Sub-Class based on ecosystem and abiotic factors:

- Systems: include Estuarine and Marine (the two important to this study) as well as Palustrine, Lacustrine, and Riverine.

- Subsystems: where applicable, Intertidal (both salt marshes and tidal flats are found here) and Subtidal.

- Class: flat, emergent, scrub/shrub (the classes important here), rocky shore, beach bar, etc.

- Subclass: includes type of vegetation, water regimes and the like. (Subclasses were not used in this analysis.)

For the resources included in this work, the inter-relationships between the various systems, classes, and subsets of each are schematically illustrated below.

\begin{tabular}{lcc} 
System & Subsystem & Class \\
\cline { 2 - 3 } Marine & Intertidal & F1at \\
Estuarine & Intertidal & F1at \\
& & Emergent Vegetation \\
& (salt marsh) \\
& & Scrub/Shrub \\
& & (shrub marsh)
\end{tabular}

An Estuarine System consists of deepwater tidal habitats and adjacent tidal wetlands that are semienclosed by land but have open, partly obstructed, or sporadic access to the open ocean, and in which ocean water is diluted by fresh water runoff from the landward side. The Estuarine System extends upstream and landward to the point that ocean-derived salinity measures less than $0.50 / 00$ (parts per thousand) during the period of average low flow. The seaward boundary is an imaginary line closing the mouth of the river, bay, or sound, or the seaward limit of emergent vegetation, whichever is greater.

The Marine System consists of the open ocean and its associated high-energy coastline. Marine habitats are exposed to the waves and currents of the open ocean. Salinities generally exceed 30 o/oo with little or no dilution except directly outside mouths of estuaries.

Emergent vegetation is characterized by erect, rooted, herbaceous, aquatic or 
water tolerant plants. This vegetation is present for most of the growing season in most years. Emergent wetlands are relative stable and retain the same appearance year after year.

Included within each polygon on the National Wetlands Inventory maps is an encoded classification of wetland type. These take the following form:

Example 1. Salt Marsh E2EM

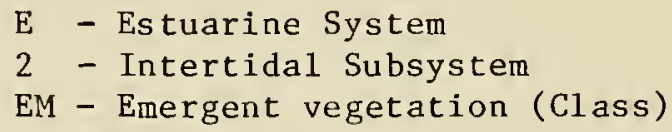

Example 2.

Shrub Marsh E2SS

$$
\begin{aligned}
& \text { E - Estuarine } \\
& 2 \text { - Intertidal Subsystem } \\
& \text { SS - Scrub/Shrub (C1ass) }
\end{aligned}
$$

Example 3 .

Estuarine Flat E 2FL

$$
\begin{aligned}
& \text { E - Estuarine System } \\
& 2 \text { - Intertidal Subsystem } \\
& \text { FL - Flat (Class) }
\end{aligned}
$$

Example 4. Marine F1at M2FL

$$
\begin{aligned}
& \text { M - Marine System } \\
& 2 \text { - Intertidal Subsystem } \\
& \text { FL - Flat (Class) }
\end{aligned}
$$

Each polygon containing these codes was identified and measured to develop the acreage figures shown in the text. Estuarine and Marine Flats were also combined to be listed as Tidal Flats. 

Property of

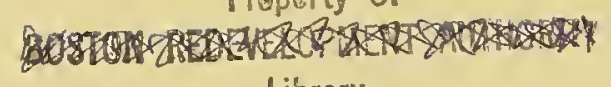

Library 
